\title{
Gender balancing staff recruitment: shortlisting
}

\section{Stewart Anderson}

It can, sometimes without fault, be all too easy to have an unconscious bias when selecting candidates for interview. Have you ever heard someone say things like: "Her application is OK although I feel I could get on better with this one as it's a man" (or of course "...a woman") or maybe even the poisonous "good application but she might want kids, so not sure"?

Shame on those individuals! They need to take time out and think about what they have done. If you're on a selection panel when this happens, make sure someone also points out what they have said and why it's wrong.

Selection of a good candidate should rarely, if ever, be based on the sex of the individual, instead we should focus on what each candidate is bringing to the table. It is their skills and previous knowledge which are relevant to the job. This is a great thought, but how can we reduce or even eradicate bias during the selection process?

\section{Ingredients}

- $\quad$ Selection panel, mixed, (optionally one or more members from outside of the team).

- Pack of Post-it notes, all the same colour, size and shape.

- Room with a table of suitable size.

- Enough pens for the panel members.
- Selection of tasty treats and beverages (non-alcoholic).

- Job description.

\section{Method}

1. Prepare a checklist by breaking down the job description into the key skills/ knowledge that you are looking for candidates to have and the level you would expect as a minimum for the position. Check that the selection panel members are familiar with the checklist (see Gender balancing staff recruitment: attracting the right candidates).

2. Anonymise the applications by covering over name, gender and other identifying demographic information. Prepare the Post-it notes by separating each on a table and then having each panel member write out "Candidate" and then the next letter in the sequence (A through to $Z$ ). If there are more than 26 candidates, add numbers 1-99 for each letter thereafter (e.g. A1, B1, $\mathrm{C}_{1} \ldots$ etc). Cover the names and other information with the Post-it notes.

3. Ask each member of the selection panel to review all of the applications, referencing the checklist as they go and taking notes of the reasons why they have picked each candidate and ensuring that each candidate is referred to by the relevant Post-it note on the application, e.g. "Candidate A". 
4. When reading recommendation letters, be aware that studies have shown that women get short-changed by their referees. A study of reference letters about medical faculty found that letters written for women medics were shorter, missed out basic features, and used language which raised doubt in the mind of the reader (Krawczyk \& Smyk, 2016). They were also inclined to associate women with their teaching and men with their research. Watch out for letters which use gendered language or weasel words or phrases like: "It appears that her health and personal life are stable" or "She has worked hard on the projects she has accepted". These are actual examples from the corpus gathered in Krawczyk \& Smyk (2016)!
5. When looking at the candidates' publications, take into account career breaks which might explain publication gaps to avoid penalising people who have taken time out to care for children and other family members.

6. Remember to feed the panel members! Being tired and hungry doesn't help with decision-making. For example, a study of judge's meal breaks found that their decisions reflected status quo just before they took a break, but were more deliberative (and lenient) after a meal break (Danziger et al., 2011).

7. Have the panel pick six candidates each and then come together as a group and agree across the panel on a final six candidates to take forward to interview. As discussed in Gender balancing staff recruitment: interviewing, it is a good idea to recruit for several posts at once.

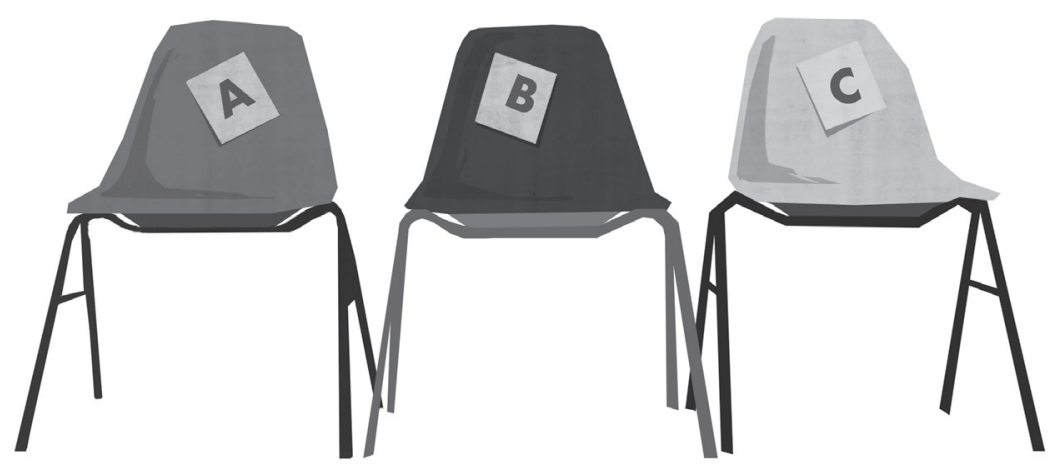

